\title{
Protracted Course of Relapsing-Remitting Fibrolamellar Hepatocellular Carcinoma in an Adolescent
}

\author{
Ankit Rao a, b, Syed Nadir Abbas ${ }^{\mathrm{a}}$, Yuk Ting Ma ${ }^{\mathrm{a}}$, Pankaj Punia ${ }^{\mathrm{a}}$
}

\begin{abstract}
Hepatocellular carcinoma (HCC) is a global healthcare problem commonest in males with hepatic cirrhosis or hepatitis B virus infection. Fibrolamellar HCC is a subtype seen mainly in younger patients without conventional risk factors and perhaps has a somewhat more favorable prognosis than typical HCC. Here we report a case of fibrolamellar HCC presenting in an adolescent with a prolonged, relapsing-remitting course characterized by prolonged exposure to sorafenib including in the adjuvant context, use of adjuvant doxorubicin chemotherapy, resection of multiple metachronous pulmonary metastases and dramatic response to radiotherapy delivered as tomotherapy to her metastatic hilar lymphadenopathy. We speculate on the possible synergistic interactions between radiotherapy and her antiangiogenic therapy and on what systemic treatment options might be open to her in the future including further targeted therapy and also immunotherapy including immune checkpoint blockade such as antiCTLA4 and PD-1.
\end{abstract}

Keywords: Fibrolamellar; Hepatocellular; Carcinoma

\section{Introduction}

Hepatocellular carcinoma (HCC) is the commonest primary epithelial neoplasm of the liver originating from hepatocytes. The majority of patients are males in late middle age with predisposing hepatic cirrhosis (of varying etiology) or chronic hepatitis B virus infection. Although a global challenge for healthcare being the fifth most common cancer worldwide, the majority of the burden of HCC falls on developing nations where endemic hepatitis B virus infection and aflatoxin exposure rather than hepatitis $\mathrm{C}$ virus and alcoholism drive most cases [1].

Manuscript accepted for publication January 29, 2015

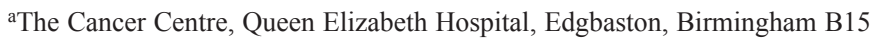
2TH, UK

${ }^{\mathrm{b} C}$ Corresponding Author: Ankit Rao, The Cancer Centre, Queen Elizabeth Hospital, Edgbaston, Birmingham B15 2TH, UK. Email: ankitrao@doctors.org.uk

doi: http://dx.doi.org/10.14740/jmc2060w
HCC in childhood is very rare and accounts for less than $0.5 \%$ of all pediatric malignancies. However, it is the second most common malignant liver neoplasm after hepatoblastoma in children. Its relative frequency is 0.5 to 1.0 cases per million children. It is commoner in adolescents than in infants. There may be some evidence that neoadjuvant chemotherapy improves the $\mathrm{R} 0$ resection rate in pediatric cases [2].

Unlike hepatoblastoma, pediatric conventional HCC usually develops in the presence of underlying liver disease and this may include chronic hepatitis B infection, metabolic disorders such as tyrosinemia, Wilson's disease [3] and glycogen storage diseases, structural problems such as biliary atresia [4] and infantile cholestatis. However, in children as compared to adults, there are more de novo cases arising in non-cirrhotic livers. The prognosis in $\mathrm{HCC}$ is poor and this may relate to the intrinsic resistance of HCC to conventional cytotoxic chemotherapy certainly compared with hepatoblastoma [5].

A subtype of HCC is fibrolamellar carcinoma. Its distinguishing features histologically include abundant fibrous collagenous bands, eosinophilic tumor cell cytoplasm and enlarged nucleoli [6]. Fibrolamellar HCC predominantly occurs in young patients without underlying hepatitis or cirrhosis. Serum $\alpha$-fetoproteins are not elevated in most cases and the male/female ratio is approximately equal. Although many early studies suggested a better prognosis with the fibrolamellar variant [7], it is increasingly being appreciated that this may well not be the case [8]. The 5-year survival rate was $76 \%$ for resected fibrolamellar HCC versus $37 \%$ for resected HCC in one study [9].

The typical presenting symptoms of HCC may include upper abdominal pain, abdominal distension, nausea and vomiting, and constitutional symptoms such as poor appetite, lack of energy and unintentional weight loss. In patients with a history of chronic liver disease, HCC may first be suspected when de-compensation of cirrhosis occurs manifesting with variceal bleeding, worsening ascites associated with peripheral oedema, jaundice and encephalopathy. HCC is also increasingly detected in asymptomatic individuals who are undergoing serological (AFP) and ultrasound surveillance in view of their liver cirrhosis or chronic hepatitis B virus infection.

Treatment for HCC is largely determined by the stage of the tumor at presentation and the Barcelona Liver Clinic staging system is widely used for this purpose. It incorporates patient-related factors such as co-morbidities, symptoms and 


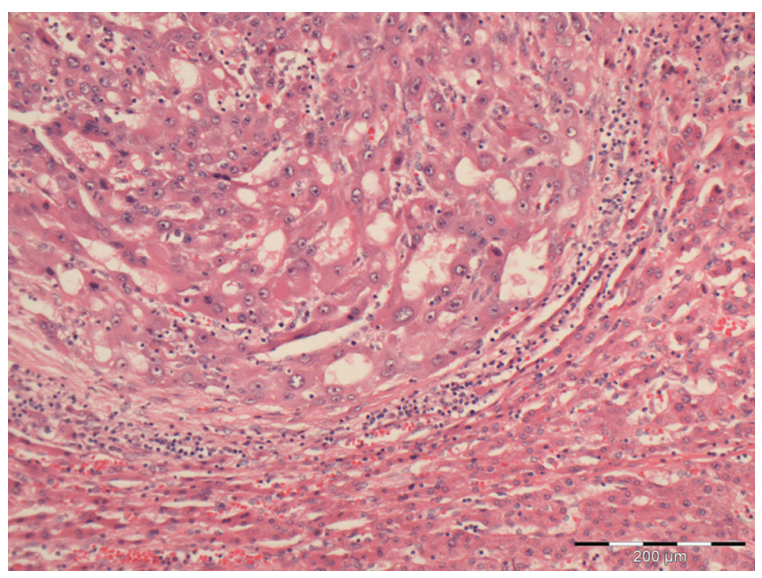

Figure 1. Histomorphological appearance of primary fibrolamellar HCC (H\&E, $\times 200)$ : top left is tumor, and bottom right is normal liver.

performance status, tumor-related factors including size and number of tumor nodules on imaging and the presence of nodal metastases and extra-hepatic spread and also the severity of any underlying cirrhosis as assessed by the Child-Pugh score and degree of portal hypertension [10]. Patients with early stage disease may be treated with curative intent with ablative techniques, partial hepatectomy or liver transplantation [11]. Intermediate stage disease is treated with loco-regional therapies including transarterial chemo-embolization (TACE) typically with doxorubicin [12] and latterly radioembolization $[13,14]$ leading to a median survival of $20-24$ months. Advanced stage HCC is treated with sorafenib, a multi-targeted small molecule tyrosine kinase inhibitor against vascular endothelial growth factor receptor, platelet-derived growth factor receptor, cKIT, RAF and Flt3 ligand, as standard-of-care. The survival benefit is demonstrable but modest with an increase of 2.8 months in median overall survival in a European population with predominantly hepatitis $\mathrm{C}$ virus infection [15] and 2.3 months in an Oriental population with mainly hepatitis B virus infection and more advanced cirrhosis [16].

\section{Case Report}

Here, we present a case of fibrolamellar HCC, diagnosed in childhood in the absence of underlying liver disease with a protracted course, multiple resections of metastatic deposits and dramatic response to radiation therapy.

A 14-year-old girl, without any significant past medical history except for meningococcal septicaemia at the age of 5 years, presented for further evaluation with a 3-month history of profound fatigue and lethargy. She also described amenorrhoea for 1 year and a recurrent rash on the bridge of her nose. She did not complain of bowel symptoms, loss of appetite, abdominal pain or jaundice.

On clinical examination, she was slightly hirsute over her trunk and was slightly tachycardic with pulse of 100 and a blood pressure of $125 / 80 \mathrm{~mm} \mathrm{Hg}$. On abdominal examination, she had an enlarged liver, $4-5 \mathrm{~cm}$ below the right costal margin. She was also hyperpigmented around her neck but no

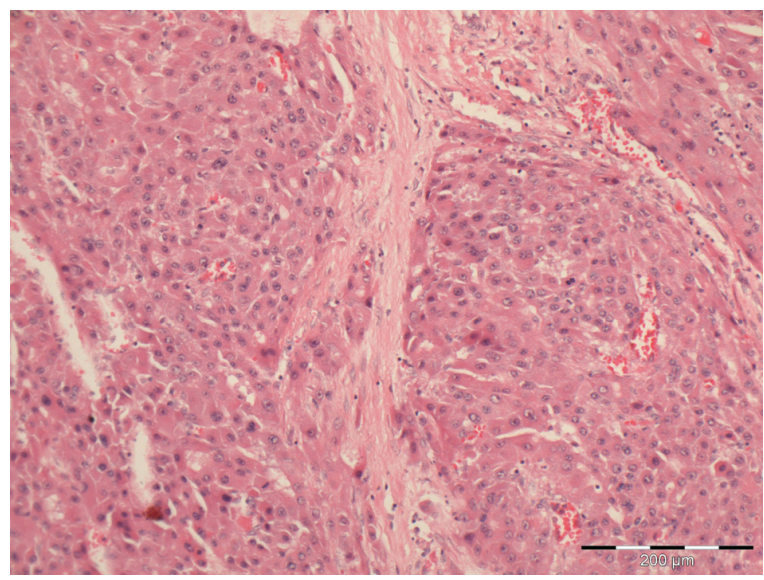

Figure 2. Classical appearance of fibrous bands within tumor: center (H\&E, $\times 200)$.

other abnormalities were noted. There was no family history of liver disease or malignancy.

Her laboratory investigations showed a slightly raised alanine aminotransferase at $51 \mathrm{IU} / \mathrm{L}$, hemoglobin of $11.2 \mathrm{~g} / \mathrm{L}$, erythrocyte sedimentation rate of $88 \mathrm{~mm} / \mathrm{h}$ and C-reactive protein of $44 \mathrm{mg} / \mathrm{L}$ with no other abnormal results. Abdominal ultrasound showed a solid echogenic lesion measuring $17 \times 18$ $\times 10 \mathrm{~cm}$ displacing hepatic and portal veins within her liver. A localized lesion was confirmed on CT imaging: there was no evidence of metastasis. Serum alpha-fetoprotein level was within the normal range.

She underwent laparotomy and extended right hemi-hepatectomy with caudate lobe resection and there was complete macroscopic clearance of visible disease. Histopathology confirmed a primary fibrolamellar HCC, measuring $28 \mathrm{~cm}$ diameter, with no histological evidence of fibrosis or steatohepatitis in the background liver and no evidence of infective or metabolic liver disease as shown in Figure 1. Figure 2 shows the typical fibrocollagenous bands of fibrolamellar HCC. Following surgery, she received adjuvant systemic therapy in the form of chemotherapy with intravenous doxorubicin and sorafenib for a period of 6 months. However, 9 months following the completion of adjuvant therapy, routine surveillance imaging detected a seemingly isolated recurrent lesion in the remnant liver. This was resected with a non-anatomical resection with clear margins and histomorphological appearances identical to the primary lesion, with evidence of focal lymphovascular invasion as shown in Figure 3. A follow-up CT scan 6 months later demonstrated multiple lung lesions. At this point, after an informed discussion with the patient and her family regarding the relative paucity of evidence for sorafenib in fibrolamellar $\mathrm{HCC}$, sorafenib was re-instituted at a dose of $800 \mathrm{mg}$ daily and this was later reduced to $600 \mathrm{mg}$ daily in view of grade $1-2$ palmar-plantar erythrodysesthesia and generalized dry skin. Over the next 3 years, she underwent multiple video-assisted thoracoscopic surgical wedge resections for asymptomatic small pulmonary nodules (one in the right lung and three in the left lung) detected on routine surveillance thoracic and abdominal imaging. Histology confirmed metastatic fibrolamellar $\mathrm{HCC}$ in all nodules (with evidence of bile production by tumor 


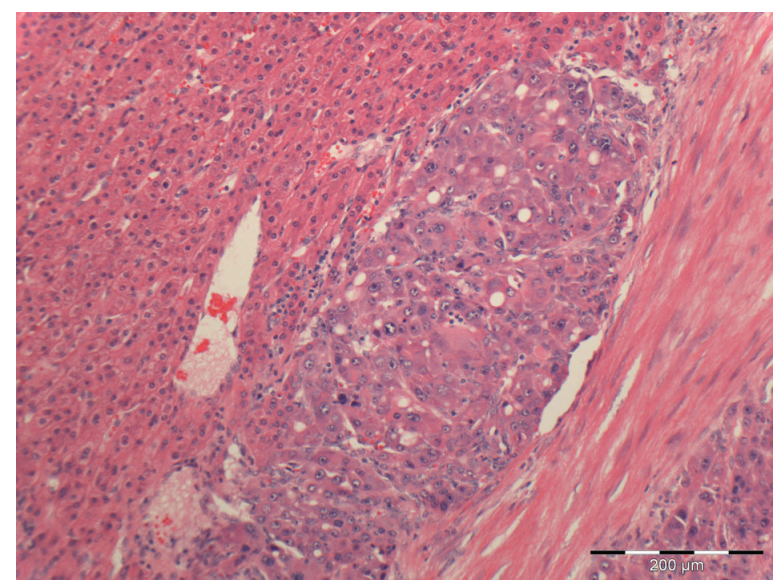

Figure 3. Vascular invasion in recurrent $\mathrm{HCC}$ lesion in liver after primary resection (H\&E, $\times 200)$. Normal liver: top left; tumor within blood vessel: center.

cells), one resected nodule had positive resection margins and a hilar lymph node was involved with tumor. Histomorphological appearances resembled those of the primary lesion in the liver (Fig. 4). She continued with sorafenib $600 \mathrm{mg}$ during this period with reasonably good tolerance.

Six months after her last VATS lung resection, imaging revealed concerning enlargement of a left hilar lymph node. This was kept under surveillance and sorafenib was continued. However, 8 months later (5 years after initial diagnosis of fibrolamellar $\mathrm{HCC}$ in the liver) there was unequivocal enlargement of the left hilar node with a new enlarged right hilar node as shown in Figure 5a, b At this point, her sorafenib dose was increased back to $800 \mathrm{mg}$ daily. Once again, a thoracic surgical opinion was sought and due to proximity of the left-sided lymph node to the inferior pulmonary vein and the right-sided node to the pulmonary artery, surgical resection was deemed inappropriate. Therefore, in a patient with an excellent performance status and no respiratory co-morbidity, an opinion regarding radical radiotherapy was sought. She underwent tomotherapy to bilateral mediastinal lymph nodes with a dose of $50 \mathrm{~Gy}$ in 20 fractions. Sorafenib was continued during radiotherapy and following completion of radiotherapy, she developed oral mucositis which had never been experienced with sorafenib previously. A follow-up CT scan 7 months after radiotherapy confirmed complete resolution of the bilateral hilar lymphadenopathy and no evidence of recurrence elsewhere (Fig. 5c, d). Six months after this, despite remaining well and free of cancer-related symptoms, she developed grade 2 palmar-plantar erythrodysasthesia despite supportive measures. Due to the adverse effects on her quality of life, after an informed discussion she discontinued sorafenib and was kept under regular surveillance. At most recent follow-up, she remains well with no clinical or radiological evidence of active cancer.

\section{Discussion}

We believe this case is highly unusual and potentially educa-

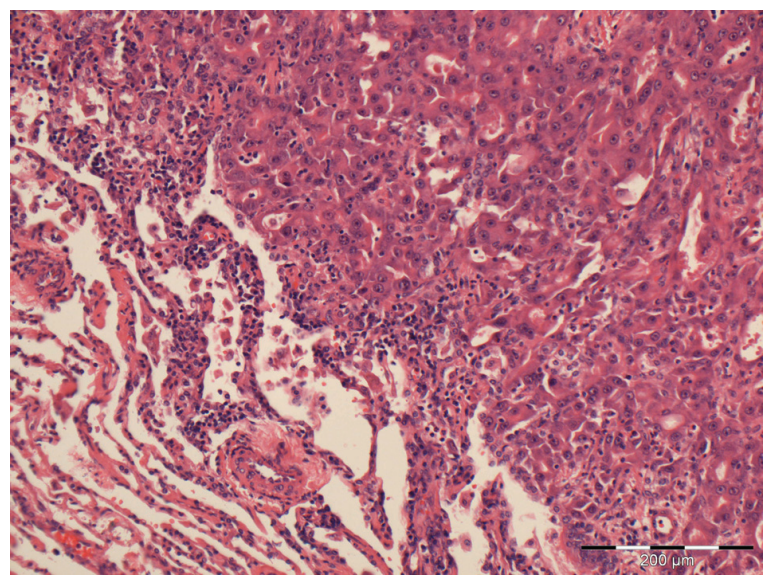

Figure 4. Histomorphological appearance of secondary metastatic deposits in lungs $(\mathrm{H} \& \mathrm{E}, \times 200)$. Top right: metastatic tumour cells; bottom left: normal alveoli.

tional in several ways including the protracted disease course with multiple metastectomies, good tolerance of sorafenib treatment over several years and the dramatic response to radiotherapy.

Although there is no evidence for chemotherapy in the adjuvant setting for resected fibrolamellar HCC, this patient received adjuvant treatment with both sorafenib and doxorubicin in view of her complete resection, young age and potential to benefit from such treatment. The evidence for efficacy of either treatment in this setting is lacking in HCC [17] although the use of sorafenib after liver resection or ablative treatment is being investigated in the STORM study of sorafenib versus placebo until recurrence or intolerance of treatment. In fibrolamellar HCC, various case series have advocated aggressive resection of recurrent lesions to improve survival of young patients [9]. Even in these cases documented median survival after second resection is in the range of $24-27$ months.

It can be speculated that since sorafenib is predominantly cytostatic, its concurrent use with chemotherapy may have blunted the potential cytotoxic effect of chemotherapy which targets dividing cells. This potential cytostatic effect of sorafenib also formed the rationale for restarting the drug when metastatic disease was detected despite its use in the adjuvant setting. The sorafenib-doxorubicin combination is currently being studied in the randomized, phase III setting for advanced HCC in the NCT01015833 study. In patients with inoperable HCC, it was reported that sorafenib and doxorubicin were tolerable, although rates of $\mathrm{G} / 3$ diarrhea and leukopenia were $17 \%$ and $45 \%$ respectively, with a disease control rate of $70 \%[18]$.

This patient underwent pulmonary metastectomies via video-assisted thoracoscopic surgery and wedge resections for metachronous oligometastatic disease in the lungs. Notably, although there was no evidence of metastatic disease outside of the thorax, the lung lesions were multiple not solitary. Again, there is a paucity of published scientific literature on this topic. Three studies, each with less than 50 patients, reported on outcomes from case series of patients who had pulmonary metastectomies for metachronous disease [19-21]. The rate of over- 

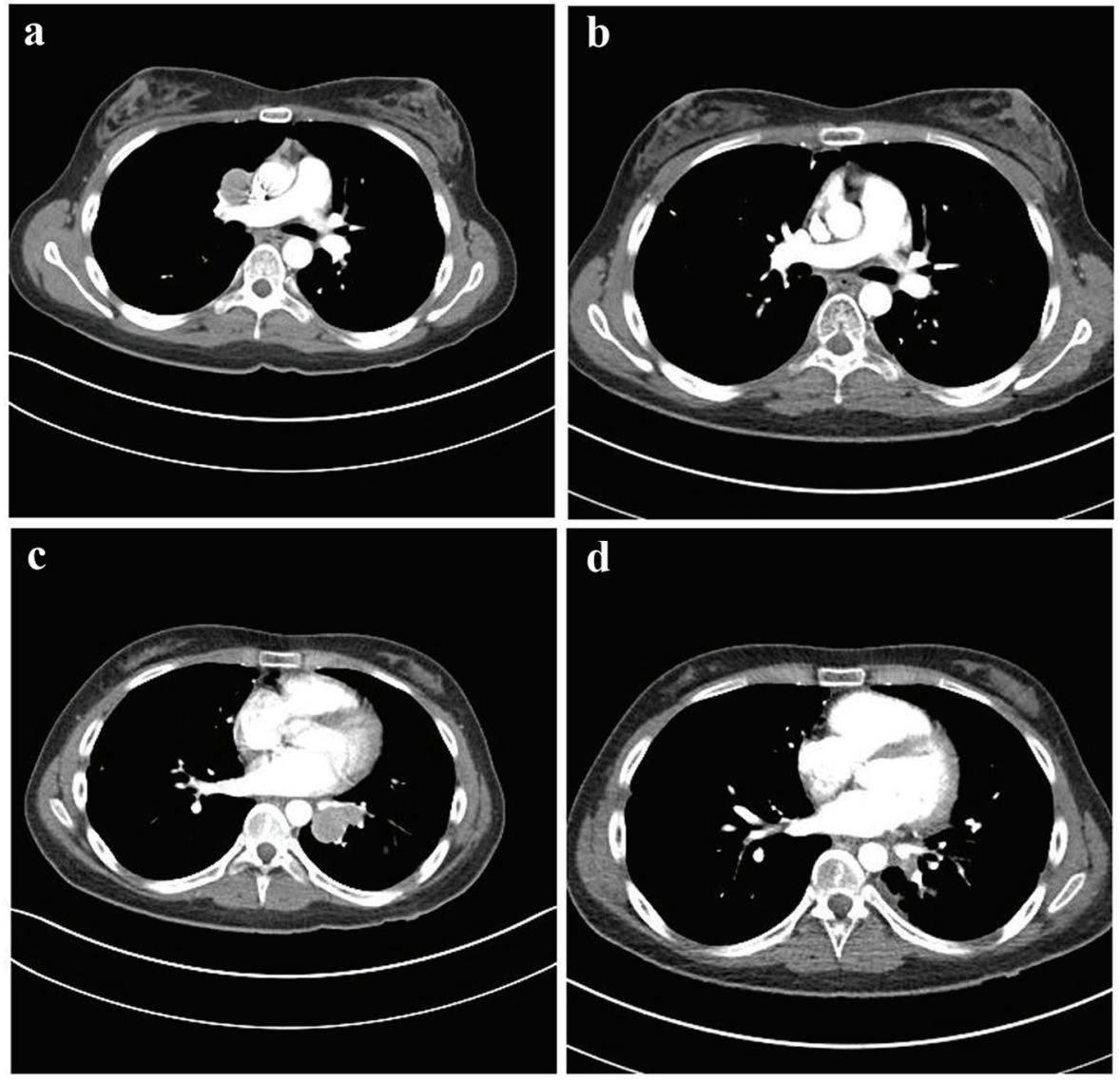

Figure 5. CT imaging of hilar lymphadenopathy. (a) Right hilar lymphadenopathy prior to radiotherapy. (b) Right hilar region post radiotherapy. (c) Left hilar lymphadenopathy prior to radiotherapy. (d) Left hilar region post radiotherapy.

all survival ranged from $24 \%$ at 3 years to $26-37 \%$ at 5 years. Solitary metastasis and longer time from resection of primary cancer to detection of metastases were associated with better survival outcomes. Clearly, such case series do not include a comparison group and therefore the favorable outcomes described may relate to intrinsically indolent disease as in this case rather than reflect any therapeutic benefit of the surgical resection.

Although HCC has long been known to be an intrinsically radiosensitive tumor, as evidenced by for example the $70-80 \%$ pain relief rate after irradiation of symptomatic skeletal metastases [22], its role in fibrolamellar HCC is unknown. Our case does demonstrate good therapeutic effect of radical dose RT in keeping with recent findings of the therapeutic efficacy of radiation therapy whether delivered externally in the form of stereotactic radiotherapy [23], sparing normal liver tissue, or internally for example with yttrium beads [14].

This patient received concurrent radiotherapy and sorafenib and whilst not an extensively studied combination in the literature, there is emerging evidence that it is a safe and feasible combination treatment [24] and, in fact, there may well be synergistic effects between anti-angiogenic therapy and radiotherapy with the former potentiating the effect of the latter [25], although this may be very dependent on dose and scheduling of radiation therapy [26].
This patient has survived for over 5 years, with evidence of local recurrent disease for 4 years and metastatic disease for 3 years and her tumor-related symptomatology has been minimal. However, having received a prolonged course of sorafenib which at best may have reduced her disease velocity and also having received 50 Gy of thoracic radiotherapy, her options for future systemic treatment are limited. These options may include further anti-angiogenic therapy with the vascular endothelial growth factor receptor 1, 2 and 3 tyrosine kinase inhibitor regorafenib, which has shown evidence of limited overall survival benefit in heavily pre-treated advanced colorectal cancer, and which is being studied for advanced sorafenib refractory HCC in the phase III, randomized, placebo controlled setting in the NCT01774344 study. Brivanib, another multi-targeted tyrosine kinase inhibitor, which has additional effects on fibroblast growth factor receptor signaling, has shown promise in advanced HCC although in the first-line setting, it was inferior to sorafenib and was less well tolerated [27]. In the post-sorafenib setting, brivanib did not improve overall survival compared to placebo but there was a radiological response rate of $10 \%$ and median time-to-progression was 1.5 months longer for brivanib than placebo [28].

Moving away from targeted therapies, immunotherapy with immune checkpoint inhibitors is emerging as a potentially highly effective therapy for cancers which were once con- 
sidered non-immunogenic such as non-small cell lung cancer [29]. In particular, there is evidence that the anti-cytotoxic-Tlymphocyte-antigen-4 (CTLA4) monoclonal antibody tremelimumab can generate potent anti-tumor and anti-viral immune responses in patients with $\mathrm{HCV}$-associated $\mathrm{HCC}$ and in the clinical setting, tremelimumab achieved a partial response rate of $18 \%$ with a median progression free survival of 6.5 months in patients with advanced stage HCC and Child-Pugh B cirrhosis [30]. Of particular relevance to this case, it is well known that radiation therapy to a particular metastatic lesion may lead to "abscopal" regression of distant (non-irradiated) lesions due to radiotherapy inducing immunogenic cell death and immune priming [31] which may be potentiated by subsequent immune checkpoint blockage.

\section{Acknowledgement}

We would like to thank Dr Rachel Brown for her histopathology review of slides and helpful comments on the manuscript.

\section{References}

1. El-Serag HB, Rudolph KL. Hepatocellular carcinoma: epidemiology and molecular carcinogenesis. Gastroenterology. 2007;132(7):2557-2576.

2. Yu SB, Kim HY, Eo H, Won JK, Jung SE, Park KW, Kim WK. Clinical characteristics and prognosis of pediatric hepatocellular carcinoma. World J Surg. 2006;30(1):4350 .

3. Iwadate H, Ohira H, Suzuki T, Abe K, Yokokawa J, Takiguchi J, Rai T, et al. Hepatocellular carcinoma associated with Wilson's disease. Intern Med. 2004;43(11):10421045 .

4. Brunati A, Feruzi Z, Sokal E, Smets F, Fervaille C, Gosseye S, Clapuyt P, et al. Early occurrence of hepatocellular carcinoma in biliary atresia treated by liver transplantation. Pediatr Transplant. 2007;11(1):117-119.

5. Haas JE, Muczynski KA, Krailo M, Ablin A, Land V, Vietti TJ, Hammond GD. Histopathology and prognosis in childhood hepatoblastoma and hepatocarcinoma. Cancer. 1989;64(5):1082-1095.

6. Liu S, Chan KW, Wang B, Qiao L. Fibrolamellar hepatocellular carcinoma. Am J Gastroenterol. 2009;104(10):26172624; quiz 2625.

7. Craig JR, Peters RL, Edmondson HA, Omata M. Fibrolamellar carcinoma of the liver: a tumor of adolescents and young adults with distinctive clinico-pathologic features. Cancer. 1980;46(2):372-379.

8. Katzenstein HM, Krailo MD, Malogolowkin MH, Ortega JA, Qu W, Douglass EC, Feusner JH, et al. Fibrolamellar hepatocellular carcinoma in children and adolescents. Cancer. 2003;97(8):2006-2012.

9. Stipa F, Yoon SS, Liau KH, Fong Y, Jarnagin WR, D'Angelica M, Abou-Alfa G, et al. Outcome of patients with fibrolamellar hepatocellular carcinoma. Cancer. 2006;106(6):1331-1338.

10. Llovet JM, Fuster J, Bruix J. The Barcelona approach: diagnosis, staging, and treatment of hepatocellular carcinoma. Liver Transpl. 2004;10(2 Suppl 1):S115-120.

11. Mazzaferro V, Regalia E, Doci R, Andreola S, Pulvirenti A, Bozzetti F, Montalto F, et al. Liver transplantation for the treatment of small hepatocellular carcinomas in patients with cirrhosis. N Engl J Med. 1996;334(11):693699.

12. Llovet JM, Bruix J. Systematic review of randomized trials for unresectable hepatocellular carcinoma: Chemoembolization improves survival. Hepatology. 2003;37(2):429442.

13. Kulik LM, Carr BI, Mulcahy MF, Lewandowski RJ, Atassi B, Ryu RK, Sato KT, et al. Safety and efficacy of 90Y radiotherapy for hepatocellular carcinoma with and without portal vein thrombosis. Hepatology. 2008;47(1):7181.

14. Salem R, Lewandowski RJ, Mulcahy MF, Riaz A, Ryu RK, Ibrahim S, Atassi B, et al. Radioembolization for hepatocellular carcinoma using Yttrium-90 microspheres: a comprehensive report of long-term outcomes. Gastroenterology. 2010;138(1):52-64.

15. Llovet JM, Ricci S, Mazzaferro V, Hilgard P, Gane E, Blanc JF, de Oliveira AC, et al. Sorafenib in advanced hepatocellular carcinoma. N Engl J Med. 2008;359(4):378-390.

16. Cheng AL, Kang YK, Chen Z, Tsao CJ, Qin S, Kim JS, Luo R, et al. Efficacy and safety of sorafenib in patients in the Asia-Pacific region with advanced hepatocellular carcinoma: a phase III randomised, double-blind, placebocontrolled trial. Lancet Oncol. 2009;10(1):25-34.

17. Schwartz JD, Schwartz M, Mandeli J, Sung M. Neoadjuvant and adjuvant therapy for resectable hepatocellular carcinoma: review of the randomised clinical trials. Lancet Oncol. 2002;3(10):593-603.

18. Richly H, Schultheis B, Adamietz IA, Kupsch P, Grubert M, Hilger RA, Ludwig M, et al. Combination of sorafenib and doxorubicin in patients with advanced hepatocellular carcinoma: results from a phase I extension trial. Eur J Cancer. 2009;45(4):579-587.

19. Kwon JB, Park K, Kim YD, Seo JH, Moon SW, Cho DG, Kim YW, et al. Clinical outcome after pulmonary metastasectomy from primary hepatocellular carcinoma: analysis of prognostic factors. World J Gastroenterol. 2008;14(37):5717-5722.

20. Nakajima J, Tanaka M, Matsumoto J, Takeuchi E, Fukami T, Takamoto S. Appraisal of surgical treatment for pulmonary metastasis from hepatocellular carcinoma. World J Surg. 2005;29(6):715-718.

21. Yoon YS, Kim HK, Kim J, Choi YS, Shim YM, Paik SW, Kim K. Long-term survival and prognostic factors after pulmonary metastasectomy in hepatocellular carcinoma. Ann Surg Oncol. 2010;17(10):2795-2801.

22. Seong J, Koom WS, Park HC. Radiotherapy for painful bone metastases from hepatocellular carcinoma. Liver Int. 2005;25(2):261-265.

23. Tse RV, Hawkins M, Lockwood G, Kim JJ, Cummings B, Knox J, Sherman M, et al. Phase I study of individualized stereotactic body radiotherapy for hepatocellular carcinoma and intrahepatic cholangiocarcinoma. J Clin Oncol. 2008;26(4):657-664. 
24. Cha J, Seong J, Lee IJ, Kim JW, Han KH. Feasibility of sorafenib combined with local radiotherapy in advanced hepatocellular carcinoma. Yonsei Med J. 2013;54(5):1178-1185.

25. Yu W, Gu K, Yu Z, Yuan D, He M, Ma N, Lai S, et al. Sorafenib potentiates irradiation effect in hepatocellular carcinoma in vitro and in vivo. Cancer Lett. 2013;329(1):109-117.

26. Kleibeuker EA, Griffioen AW, Verheul HM, Slotman BJ, Thijssen VL. Combining angiogenesis inhibition and radiotherapy: a double-edged sword. Drug Resist Updat. 2012;15(3):173-182.

27. Johnson PJ, Qin S, Park JW, Poon RT, Raoul JL, Philip PA, Hsu $\mathrm{CH}$, et al. Brivanib versus sorafenib as first-line therapy in patients with unresectable, advanced hepatocellular carcinoma: results from the randomized phase III BRISK-FL study. J Clin Oncol. 2013;31(28):3517-3524.

28. Llovet JM, Decaens T, Raoul JL, Boucher E, Kudo M,
Chang C, Kang YK, et al. Brivanib in patients with advanced hepatocellular carcinoma who were intolerant to sorafenib or for whom sorafenib failed: results from the randomized phase III BRISK-PS study. J Clin Oncol. 2013;31(28):3509-3516.

29. Topalian SL, Hodi FS, Brahmer JR, Gettinger SN, Smith DC, McDermott DF, Powderly JD, et al. Safety, activity, and immune correlates of anti-PD-1 antibody in cancer. $\mathrm{N}$ Engl J Med. 2012;366(26):2443-2454.

30. Sangro B, Gomez-Martin C, de la Mata M, Inarrairaegui M, Garralda E, Barrera P, Riezu-Boj JI, et al. A clinical trial of CTLA-4 blockade with tremelimumab in patients with hepatocellular carcinoma and chronic hepatitis C. J Hepatol. 2013;59(1):81-88.

31. Ohba K, Omagari K, Nakamura T, Ikuno N, Saeki S, Matsuo I, Kinoshita H, et al. Abscopal regression of hepatocellular carcinoma after radiotherapy for bone metastasis. Gut. 1998;43(4):575-577. 